Research Article

\title{
Infinitely Many Periodic Solutions for Nonautonomous Sublinear Second-Order Hamiltonian Systems
}

\author{
Peng Zhang ${ }^{1}$ and Chun-Lei Tang ${ }^{2}$ \\ ${ }^{1}$ Department of Mathematics, ZunYi Normal College, ZunYi 563002, China \\ ${ }^{2}$ Department of Mathematics, School of Mathematics and Statistics, Southwest University, \\ Chongqing 400715, China
}

Correspondence should be addressed to Peng Zhang, gzzypd@sina.com

Received 6 February 2010; Accepted 7 June 2010

Academic Editor: Jean Mawhin

Copyright (C) 2010 P. Zhang and C.-L. Tang. This is an open access article distributed under the Creative Commons Attribution License, which permits unrestricted use, distribution, and reproduction in any medium, provided the original work is properly cited.

Two sequences of distinct periodic solutions for second-order Hamiltonian systems with sublinear nonlinearity are obtained by using the minimax methods. One sequence of solutions is local minimum points of functional, and the other is minimax type critical points of functional. We do not assume any symmetry condition on nonlinearity.

\section{Introduction and Main Result}

We are interested in the following second order Hamiltonian systems:

$$
\begin{gathered}
\ddot{u}(t)+\nabla F(t, u(t))=0 \quad \text { a.e. } t \in[0, T], \\
u(0)-u(T)=\dot{u}(0)-\dot{u}(T)=0,
\end{gathered}
$$

where $T>0$ and $F:[0, T] \times R^{N} \rightarrow R$ satisfies the following assumption.

(A) $F(t, x)$ is measurable in $t$ for each $x \in R^{N}$ and continuously differentiable in $x$ for a.e. $t \in[0, T], F(t, 0)=0$ for a.e. $t \in[0, T]$, and there exist $a \in C\left(R^{+}, R^{+}\right), b \in$ $L^{1}\left(0, T ; R^{+}\right)$such that

$$
|F(t, x)| \leq a(|x|) b(t), \quad|\nabla F(t, x)| \leq a(|x|) b(t)
$$

for all $x \in R^{N}$ and a.e. $t \in[0, T]$. 
Then the corresponding functional $\varphi$ on $H_{T}^{1}$ given by

$$
\varphi(u)=\frac{1}{2} \int_{0}^{T}|\dot{u}(t)|^{2} d t-\int_{0}^{T} F(t, u(t)) d t
$$

is continuously differentiable and weakly lower semicontinuous on $H_{T}^{1}$, where

$$
H_{T}^{1}=\left\{u:[0, T] \longrightarrow R^{N} \mid u \text { is absolutely continuous, } u(0)=u(T), \dot{u} \in L^{2}\left(0, T ; R^{N}\right)\right\}
$$

is a Hilbert space with the norm defined by

$$
\|u\|=\left(\int_{0}^{T}|u(t)|^{2} d t+\int_{0}^{T}|\dot{u}(t)|^{2} d t\right)^{1 / 2}
$$

for $u \in H_{T}^{1}$ (see [1]). Moreover,

$$
\left\langle\varphi^{\prime}(u), v\right\rangle=\int_{0}^{T}(\dot{u}(t), \dot{v}(t)) d t-\int_{0}^{T}(\nabla F(t, u(t)), v(t)) d t
$$

for all $u, v \in H_{T}^{1}$. It is well known that the solutions of problem (1.1) correspond to the critical points of $\varphi$.

There are large number of papers that deal with multiplicity results for this problem. Infinitely many solutions for problem (1.1) are obtained in [2-4], where the symmetry assumption on the nonlinearity $F$ has played an important role. In recent years, many authors have paid much attention to weaken the symmetry condition, and some existence results on periodic and subharmonic solutions have been obtained without the symmetry condition (see [5-7]). Particularly, Ma and Zhang [6] got the existence of a sequence of distinct periodic solutions under some superquadratic and asymptotic quadratic cases. Faraci and Livrea [7] studied the existence of infinitely many periodic solutions under the assumption that $F(t, x)$ is a suitable oscillating behaviour either at infinity or at zero.

In this paper, we suppose that the nonlinearity $\nabla F(t, x)$ is sublinear, that is, there exist $f, g \in L^{1}\left(0, T ; R^{+}\right)$and $\alpha \in[0,1]$ such that

$$
|\nabla F(t, x)| \leq f(t)|x|^{\alpha}+g(t)
$$

for all $x \in R^{N}$ and a.e. $t \in[0, T]$. We establish some multiplicity results for problem (1.1) under different assumptions on the potential $F$. Roughly speaking, we assume that $F$ has a suitable oscillating behaviour at infinity. Two sequences of distinct periodic solutions are obtained by using the minimax methods. One sequence of solutions is local minimum points of functional, and the other is minimax type critical points of functional. In particular, we do not assume any symmetry condition at all. 
Our main result is the following theorem.

Theorem 1.1. Suppose that $F(t, x)$ satisfies assumptions (A) and (1.7). Assume that

$$
\begin{aligned}
& \limsup _{r \rightarrow+\infty} \inf _{x \in R^{N},|x|=r}|x|^{-2 \alpha} \int_{0}^{T} F(t, x) d t=+\infty, \\
& \liminf _{R \rightarrow+\infty} \sup _{x \in R^{N},|x|=R}|x|^{-2 \alpha} \int_{0}^{T} F(t, x) d t=-\infty .
\end{aligned}
$$

Then,

(i) there exists a sequence of periodic solutions $\left\{u_{n}\right\}$ which are minimax type critical points of functional $\varphi$, and $\varphi\left(u_{n}\right) \rightarrow+\infty$, as $n \rightarrow \infty$;

(ii) there exists another sequence of periodic solutions $\left\{u_{m}^{*}\right\}$ which are local minimum points of functional $\varphi$, and $\varphi\left(u_{m}^{*}\right) \rightarrow-\infty$, as $m \rightarrow \infty$.

\section{Proof of Theorems}

For $u \in H_{T}^{1}$, let $\bar{u}=(1 / T) \int_{0}^{T} u(t) d t$ and $\tilde{u}=u-\bar{u}$. Then one has

$$
\begin{gathered}
\|\tilde{u}\|_{\infty}^{2} \leq \frac{T}{12} \int_{0}^{T}|\dot{u}(t)|^{2} d t \quad \text { (Sobolev's inequality) } \\
\int_{0}^{T}|\tilde{u}(t)|^{2} d t \leq \frac{T^{2}}{4 \pi^{2}} \int_{0}^{T}|\dot{u}(t)|^{2} d t \quad \text { (Wirtinger's inequality). }
\end{gathered}
$$

Lemma 2.1. Let $\widetilde{H}_{T}^{1}$ be the subspace of $H_{T}^{1}$ given by

$$
\widetilde{H}_{T}^{1}=\left\{u \in H_{T}^{1} \mid \bar{u}=0\right\}
$$

Suppose that (1.7) holds. Then

$$
\varphi(u) \longrightarrow+\infty
$$

as $\|u\| \rightarrow \infty$ in $\widetilde{H}_{T}^{1}$. 
Proof. It follows from (1.7) and Sobolev's inequality that

$$
\begin{aligned}
\varphi(u) & =\frac{1}{2} \int_{0}^{T}|\dot{u}(t)|^{2} d t-\int_{0}^{T} F(t, u(t)) d t \\
& \geq \frac{1}{2} \int_{0}^{T}|\dot{u}(t)|^{2} d t-\int_{0}^{T} f(t)|u(t)|^{\alpha+1} d t-\int_{0}^{T} g(t)|u(t)| d t \\
& \geq \frac{1}{2} \int_{0}^{T}|\dot{u}(t)|^{2} d t-\|u\|_{\infty}^{\alpha+1} \int_{0}^{T} f(t) d t-\|u\|_{\infty} \int_{0}^{T} g(t) d t \\
& \geq \frac{1}{2} \int_{0}^{T}|\dot{u}(t)|^{2} d t-C_{1}\left(\int_{0}^{T}|\dot{u}(t)|^{2} d t\right)^{(\alpha+1) / 2}-C_{2}\left(\int_{0}^{T}|\dot{u}(t)|^{2} d t\right)^{1 / 2}
\end{aligned}
$$

for all $u$ in $\widetilde{H}_{T}^{1}$. By Wirtinger's inequality, the norm

$$
\|u\|=\left(\int_{0}^{T}|\dot{u}(t)|^{2} d t\right)^{1 / 2}
$$

is an equivalent norm on $\widetilde{H}_{T}^{1}$. Hence the lemma follows from the equivalence and the above inequality.

Lemma 2.2. Suppose that (1.7) and (1.8) hold. Then there exists positive real sequence $\left\{a_{n}\right\}$ such that

$$
\begin{gathered}
\lim _{n \rightarrow \infty} a_{n}=+\infty \\
\lim _{n \rightarrow \infty} \sup _{u \in R^{N},|u|=a_{n}} \varphi(u)=-\infty
\end{gathered}
$$

The proof of this lemma is similar to the following lemma.

Lemma 2.3. Suppose that (1.7) and (1.9) hold. Then there exists positive real sequence $\left\{b_{m}\right\}$ such that

$$
\begin{gathered}
\lim _{m \rightarrow \infty} b_{m}=+\infty, \\
\lim _{m \rightarrow \infty} \inf _{u \in H_{b_{m}}} \varphi(u)=+\infty,
\end{gathered}
$$

where $H_{b_{m}}=\left\{x \in R^{N}:|u|=b_{m}\right\} \oplus \widetilde{H}_{T}^{1}$. 
Proof. For any $u \in H_{b_{m}}$, let $u=\bar{u}+\widetilde{u}$, where $|\bar{u}|=b_{m}, \tilde{u} \in \widetilde{H}_{T}^{1}$. It follows from (1.7) and Sobolev's inequality that

$$
\begin{aligned}
& \left|\int_{0}^{T}[F(t, u(t))-F(t, \bar{u})] d t\right| \\
& \quad=\left|\int_{0}^{T} \int_{0}^{1}(\nabla F(t, \bar{u}+s \tilde{u}(t)), \tilde{u}(t)) d s d t\right| \\
& \quad \leq \int_{0}^{T} \int_{0}^{1} f(t)|\bar{u}+s \tilde{u}(t)|^{\alpha}|\tilde{u}(t)| d s d t+\int_{0}^{T} \int_{0}^{1} g(t)|\tilde{u}(t)| d s d t \\
& \quad \leq 2\left(|\bar{u}|^{\alpha}+\|\tilde{u}\|_{\infty}^{\alpha}\right)\|\tilde{u}\|_{\infty} \int_{0}^{T} f(t) d t+\|\tilde{u}\|_{\infty} \int_{0}^{T} g(t) d t \\
& \quad \leq \frac{3}{T}\|\tilde{u}\|_{\infty}^{2}+\frac{T}{3}|\bar{u}|^{2 \alpha}\left(\int_{0}^{T} f(t) d t\right)^{2}+2\|\tilde{u}\|_{\infty}^{\alpha+1} \int_{0}^{T} f(t) d t+\|\tilde{u}\|_{\infty} \int_{0}^{T} g(t) d t \\
& \quad \leq \frac{1}{4} \int_{0}^{T}|\dot{u}(t)|^{2} d t+C_{1}|\bar{u}|^{2 \alpha}+C_{2}\left(\int_{0}^{T}|\dot{u}(t)|^{2} d t\right)^{(\alpha+1) / 2}+C_{3}\left(\int_{0}^{T}|\dot{u}(t)|^{2} d t\right)^{1 / 2},
\end{aligned}
$$

for all $u \in H_{T}^{1}$. Hence we have

$$
\begin{aligned}
\varphi(u)= & \frac{1}{2} \int_{0}^{T}|\dot{u}(t)|^{2} d t-\int_{0}^{T}[F(t, u(t))-F(t, \bar{u})] d t-\int_{0}^{T} F(t, \bar{u}) d t \\
\geq & \frac{1}{4} \int_{0}^{T}|\dot{u}(t)|^{2} d t-C_{2}\left(\int_{0}^{T}|\dot{u}(t)|^{2} d t\right)^{(\alpha+1) / 2}-C_{3}\left(\int_{0}^{T}|\dot{u}(t)|^{2} d t\right)^{1 / 2} \\
& -|\bar{u}|^{2 \alpha}\left(|\bar{u}|^{-2 \alpha} \int_{0}^{T} F(t, \bar{u}) d t+C_{1}\right)
\end{aligned}
$$

for all $u \in H_{T}^{1}$. As $\left(|\bar{u}|^{2}+\|\dot{u}(t)\|_{L^{2}}\right)^{1 / 2} \rightarrow \infty$ if and only if $\|u\| \rightarrow \infty$, then the lemma follows from (1.9) and the above inequality.

Now we give the proof Theorem 1.1.

Proof of Theorem 1.1. Let $B_{a_{n}}$ be a ball in $R^{N}$ with radius $a_{n}$. Define

$$
\begin{gathered}
S_{n}=\left\{\gamma \in C\left(B_{a_{n}}, H_{T}^{1}\right),\left.r\right|_{\partial B_{a_{n}}}=\left.\mathrm{id}\right|_{\partial B_{a_{n}}}\right\}, \\
c_{n}=\inf _{\gamma \in S_{n}}\left[\max _{x \in B_{a_{n}}} \varphi(\gamma(x))\right] .
\end{gathered}
$$


We claim that each $\gamma$ intersects the hyperplane $\widetilde{H}_{T}^{1}$. In fact, let $\pi: H_{T}^{1} \rightarrow R^{N}$ be the projection of $H_{T}^{1}$ onto $R^{N}$, defined by

$$
\pi(u)=\frac{1}{T} \int_{0}^{T} u(t) d t
$$

For $t \in[0,1], u \in R^{N}$, define

$$
\gamma_{t}(u)=t \pi(\gamma(u))+(1-t) u
$$

Then $\gamma_{t} \in C\left(R^{N} ; R^{N}\right)$ is a homotopy of $\gamma_{0}=$ id with $\gamma_{1}=\pi \circ \gamma$. Moreover, $\left.\gamma_{t}\right|_{\partial B_{a_{n}}}=\mathrm{id}$ for all $t \in[0,1]$. By homotopy invariance and normalization of the degree, we have

$$
\operatorname{deg}\left(\pi \circ \gamma, B_{a_{n}}, 0\right)=\operatorname{deg}\left(\mathrm{id}, B_{a_{n}}, 0\right)=1,
$$

which means that $0 \in \pi\left(\gamma\left(B_{a_{n}}\right)\right)$. Thus $\gamma\left(B_{a_{n}}\right)$ intersects the hyperplane $\widetilde{H}_{T}^{1}$.

By Lemma 2.1, the functional $\varphi$ is coercive on $\widetilde{H}_{T}^{1}$. There is a constant $M$ such that

$$
\max _{x \in B_{a_{n}}} \varphi(\gamma(x)) \geq \inf _{u \in \widetilde{H}_{T}^{1}} \varphi(u) \geq M
$$

Hence $c_{n} \geq M$. In view of Lemma 2.2, for all large values of $n$,

$$
c_{n}>\max _{x \in R^{N},|x|=a_{n}} \varphi(x)
$$

For such $n$, there exists a sequence $\left\{\gamma_{k}\right\}$ in $S_{n}$ such that

$$
\max _{x \in B_{a_{n}}} \varphi\left(\gamma_{k}(x)\right) \longrightarrow c_{n}, \quad k \longrightarrow \infty
$$

Applying Theorem 4.3 and Corollary 4.3 in [1], there exists a sequence $\left\{v_{k}\right\}$ in $H_{T}^{1}$ such that

$$
\begin{gathered}
\varphi\left(v_{k}\right) \longrightarrow c_{n}, \\
\operatorname{dist}\left(v_{k}, \gamma_{k}\left(B_{a_{n}}\right)\right) \longrightarrow 0, \\
\varphi^{\prime}\left(v_{k}\right) \longrightarrow 0,
\end{gathered}
$$

as $k \rightarrow \infty$.

Now, let us prove that the sequence $\left\{v_{k}\right\}$ is bounded in $H_{T}^{1}$. For any large enough $k$,

$$
c_{n} \leq \max _{x \in B_{a_{n}}} \varphi\left(\gamma_{k}(x)\right) \leq c_{n}+1
$$


we can find $w_{k} \in \gamma_{k}\left(B_{a_{n}}\right)$, such that

$$
\left\|v_{k}-w_{k}\right\| \leq 1
$$

For fixed $n$, by Lemma 2.3, we can choose $m$ such that $b_{m}>a_{n}$ and $\gamma_{k}\left(B_{a_{n}}\right)$ cannot intersect the hyperplane $H_{b_{m}}$. Let $w_{k}=\bar{w}_{k}+\widetilde{w}_{k}$, where $\bar{w}_{k} \in R^{N}$ and $\widetilde{w}_{k} \in \widetilde{H}_{T}^{1}$. Then we have

$$
\left|\bar{w}_{k}\right|=\left|\frac{1}{T} \int_{0}^{T} w_{k}(t) d t\right| \leq \frac{1}{T} \int_{0}^{T}\left|w_{k}(t)\right| d t \leq\left\|w_{k}\right\|_{\infty}=\max _{t \in[0, T]}\left|w_{k}(t)\right|<b_{m},
$$

for $b_{m}$ large enough. Besides, by Sobolev's inequality and (1.7), it is obvious that

$$
\begin{aligned}
c_{n}+1 \geq & \varphi\left(w_{k}\right)=\frac{1}{2} \int_{0}^{T}\left|\dot{w}_{k}(t)\right|^{2} d t-\int_{0}^{T} F\left(t, w_{k}(t)\right) d t \\
\geq & \frac{1}{2} \int_{0}^{T}\left|\dot{w}_{k}(t)\right|^{2} d t-\int_{0}^{T} f(t)\left|w_{k}(t)\right|^{1+\alpha} d t-\int_{0}^{T} g(t)\left|w_{k}(t)\right| d t \\
\geq & \frac{1}{2} \int_{0}^{T}\left|\dot{w}_{k}(t)\right|^{2} d t-4 \int_{0}^{T} f(t)\left(\left|\bar{w}_{k}(t)\right|^{1+\alpha}+\left|\tilde{w}_{k}(t)\right|^{1+\alpha}\right) d t \\
& -\int_{0}^{T} g(t)\left(\left|\bar{w}_{k}(t)\right|+\left|\tilde{w}_{\mathrm{k}}(t)\right|\right) d t \\
= & \frac{1}{2} \int_{0}^{T}\left|\dot{w}_{k}(t)\right|^{2} d t-C_{1}\left(\int_{0}^{T}\left|\dot{w}_{k}(t)\right|^{2} d t\right)^{(1+\alpha) / 2}-C_{2}\left(\int_{0}^{T}\left|\dot{w}_{k}(t)\right|^{2} d t\right)^{1 / 2}-C_{3} .
\end{aligned}
$$

As $\left(|\bar{u}|^{2}+\|\dot{u}(t)\|_{L^{2}}\right)^{1 / 2}$ is an equivalent norm in $H_{T}^{1}$, it follows that $\widetilde{w}_{k}(t)$ is bounded. Hence, $w_{k}$ is bounded. Also $\left\{v_{k}\right\}$ is bounded in $H_{T}^{1}$.

We assume that

$$
\begin{gathered}
v_{k} \rightarrow u_{n} \quad \text { weakly in } H_{T}^{1}, \\
v_{k} \longrightarrow u_{n} \quad \text { uniformly in } C\left([0, T] ; R^{N}\right),
\end{gathered}
$$

hence

$$
\begin{gathered}
\left\langle\varphi^{\prime}\left(v_{k}\right)-\varphi^{\prime}\left(u_{n}\right), v_{k}-u_{n}\right\rangle \longrightarrow 0, \\
\int_{0}^{T}\left(\nabla F\left(t, v_{k}\right)-\nabla F\left(t, u_{n}\right), v_{k}-u_{n}\right) d t \longrightarrow 0
\end{gathered}
$$

as $k \rightarrow \infty$. Moreover, an easy computation shows that

$$
\left\langle\varphi^{\prime}\left(v_{k}\right)-\varphi^{\prime}\left(u_{n}\right), v_{k}-u_{n}\right\rangle=\left\|\dot{v}_{k}-\dot{u}_{n}\right\|_{L^{2}}^{2}-\int_{0}^{T}\left(\nabla F\left(t, v_{k}\right)-\nabla F\left(t, u_{n}\right), v_{k}-u_{n}\right) d t
$$


so $\left\|\dot{v}_{k}-\dot{u}_{n}\right\|_{L^{2}} \rightarrow 0$ as $n \rightarrow \infty$. Then, it is not difficult to obtain $\left\|v_{k}-u_{n}\right\| \rightarrow 0$, as $k \rightarrow \infty$.

Now we have

$$
\begin{aligned}
& \varphi^{\prime}\left(u_{n}\right)=\lim _{k \rightarrow \infty} \varphi^{\prime}\left(v_{k}\right)=0, \\
& \varphi\left(u_{n}\right)=\lim _{k \rightarrow \infty} \varphi\left(v_{k}\right)=c_{n} .
\end{aligned}
$$

Thus, $u_{n}$ is critical point and $c_{n}$ is critical value of functional $\varphi$. For any $\gamma \in S_{n}$, if $a_{n}>b_{m}$, $\gamma\left(B_{a_{n}}\right)$ intersects the hyperplane $H_{b_{m}}=\left\{x \in R^{N},|u|=b_{m}\right\} \oplus \widetilde{H}_{T}^{1}$. It follows that

$$
\max _{x \in B_{a_{n}}} \varphi(\gamma(x)) \geq \inf _{u \in H_{b_{m}}} \varphi(u)
$$

This inequality and Lemma 2.3 deduce that

$$
\lim _{n \rightarrow \infty} c_{n}=+\infty
$$

The first result of Theorem 1.1 is obtained.

For fixed $m$, define the subset $P_{m}$ of $H_{T}^{1}$ by

$$
P_{m}=\left\{u \in H_{T}^{1}: u=\bar{u}+\widetilde{u},|\bar{u}| \leq b_{m}, \widetilde{u} \in \widetilde{H}_{T}^{1}\right\} .
$$

For $u \in P_{m}$, we have

$$
\begin{aligned}
\varphi(u)= & \frac{1}{2} \int_{0}^{T}|\dot{u}(t)|^{2} d t-\int_{0}^{T} F(t, u(t)) d t \\
\geq & \frac{1}{2} \int_{0}^{T}|\dot{u}(t)|^{2} d t-\int_{0}^{T} f(t)|u(t)|^{1+\alpha} d t-\int_{0}^{T} g(t)|u(t)| d t \\
\geq & \frac{1}{2} \int_{0}^{T}|\dot{u}(t)|^{2} d t-4 \int_{0}^{T} f(t)\left(\left|\bar{w}_{k}(t)\right|^{1+\alpha}+|\tilde{u}(t)|^{1+\alpha}\right) d t \\
& -\int_{0}^{T} g(t)(|\bar{u}(t)|+|\tilde{u}(t)|) d t \\
= & \frac{1}{2} \int_{0}^{T}|\dot{u}(t)|^{2} d t-C_{4}\left(\int_{0}^{T}|\dot{u}(t)|^{2} d t\right)^{(1+\alpha) / 2}-C_{5}\left(\int_{0}^{T}|\dot{u}(t)|^{2} d t\right)^{1 / 2}-C_{6} .
\end{aligned}
$$

It follows that $\varphi$ is bounded below on $P_{m}$. Define

$$
\mu_{m}=\inf _{u \in P_{m}} \varphi(u),
$$


and let $\left\{u_{k}\right\}$ be a minimizing sequence in $P_{m}$, that is,

$$
\varphi\left(u_{k}\right) \longrightarrow \mu_{m} \quad \text { as } k \longrightarrow \infty
$$

From (2.29), $\left\{u_{k}\right\}$ is bounded in $H_{T}^{1}$. Then, there is a subsequence, we also denoted by $\left\{u_{k}\right\}$, such that

$$
u_{k} \rightarrow u_{m}^{*} \quad \text { weakly in } H_{T}^{1} \text {. }
$$

The case that $P_{m}$ is a convex closed subset of $H_{T}^{1}$ implies that $u_{m}^{*} \in P_{m}$.As $\varphi$ is weakly lower semicontinuous, we have

$$
\mu_{m}=\lim _{k \rightarrow \infty} \varphi\left(u_{k}\right) \geq \varphi\left(u_{m}^{*}\right)
$$

Since $u_{m}^{*} \in P_{m}$,

$$
\mu_{m}=\varphi\left(u_{m}^{*}\right)
$$

Suppose that $u_{m}^{*}$ is in the interior of $P_{m}$, then $u_{m}^{*}$ is a local minimum of functional $\varphi$. In fact, let $u_{m}^{*}=\bar{u}_{m}^{*}+\widetilde{u}_{m}^{*}$. For large $m$, from Lemmas 2.2 and 2.3, we have $\left|\bar{u}_{m}^{*}\right| \neq b_{m}$, which means that $u_{m}^{*}$ is not on the boundary of $P_{m}$.

Finally, as $u_{m}^{*}$ is a minimum of $\varphi$ in $P_{m}$,

$$
\varphi\left(u_{m}^{*}\right) \leq \sup _{|x|=b_{m}} \varphi(x)
$$

It follows from Lemma 2.2 that

$$
\varphi\left(u_{m}^{*}\right)=-\infty
$$

Therefore Theorem 1.1 is proved.

\section{Acknowledgments}

This work is supported by NNSF of China under Grant 10771173 and Natural Science Foundation of Education of Guizhou Province under Grant 2008067.

\section{References}

[1] J. Mawhin and M. Willem, Critical Point Theory and Hamiltonian Systems, vol. 74 of Applied Mathematical Sciences, Springer, New York, NY, USA, 1989.

[2] F. Antonacci and P. Magrone, "Second order nonautonomous systems with symmetric potential changing sign," Rendiconti di Matematica e delle sue Applicazioni. Serie VII, vol. 18, no. 2, pp. 367-379, 1998. 
[3] X. P. Wu and C. L. Tang, "Periodic solutions of nonautonomous second-order Hamiltonian systems with even-typed potentials," Nonlinear Analysis: Theory, Methods \& Applications, vol. 55, no. 6, pp. 759769, 2003.

[4] W. M. Zou and S. J. Li, "Infinitely many solutions for Hamiltonian systems," Journal of Differential Equations, vol. 186, no. 1, pp. 141-164, 2002.

[5] Z.-L. Tao and C.-L. Tang, "Periodic and subharmonic solutions of second-order Hamiltonian systems," Journal of Mathematical Analysis and Applications, vol. 293, no. 2, pp. 435-445, 2004.

[6] S. W. Ma and Y. X. Zhang, "Existence of infinitely many periodic solutions for ordinary $p$-Laplacian systems," Journal of Mathematical Analysis and Applications, vol. 351, no. 1, pp. 469-479, 2009.

[7] F. Faraci and R. Livrea, "Infinitely many periodic solutions for a second-order nonautonomous system," Nonlinear Analysis: Theory, Methods E Applications, vol. 54, no. 3, pp. 417-429, 2003. 


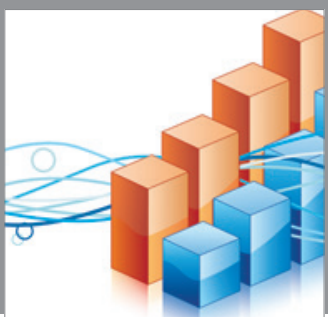

Advances in

Operations Research

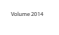

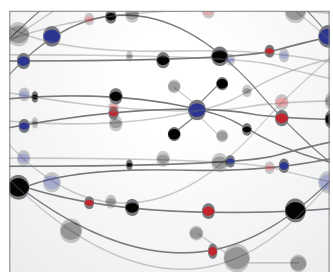

\section{The Scientific} World Journal
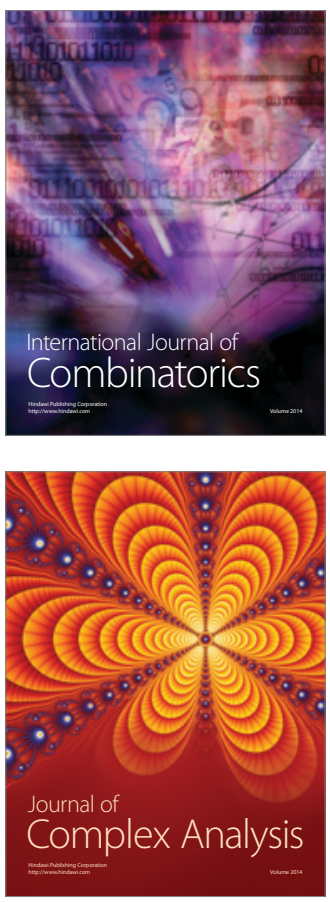

International Journal of

Mathematics and

Mathematical

Sciences
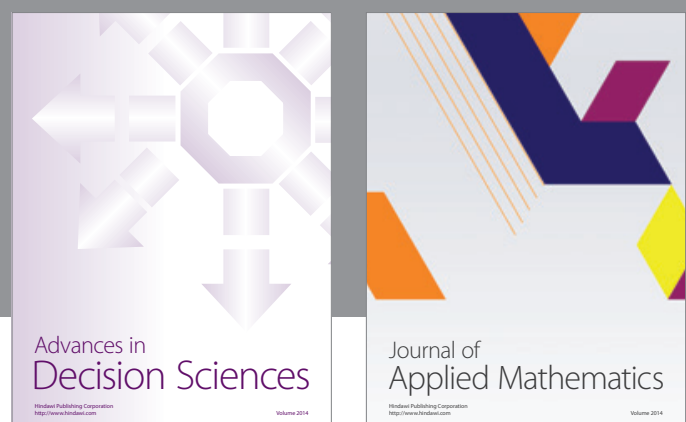

Journal of

Applied Mathematics
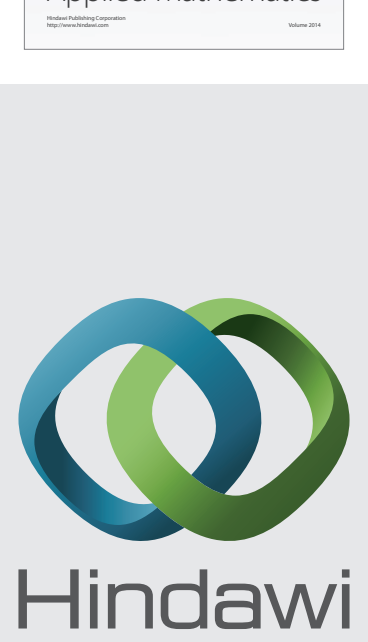

Submit your manuscripts at http://www.hindawi.com
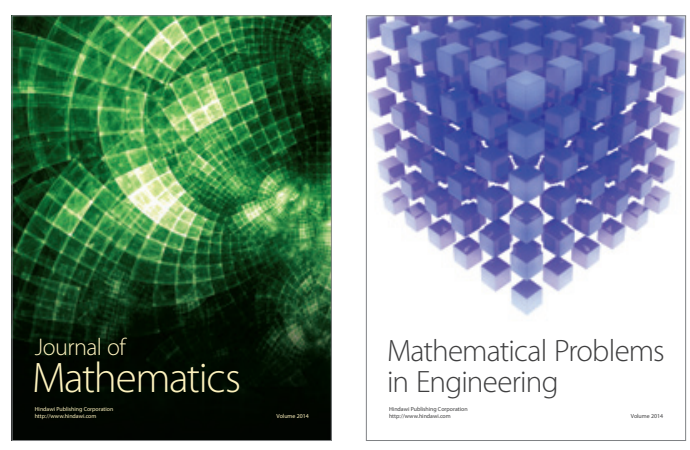

Mathematical Problems in Engineering
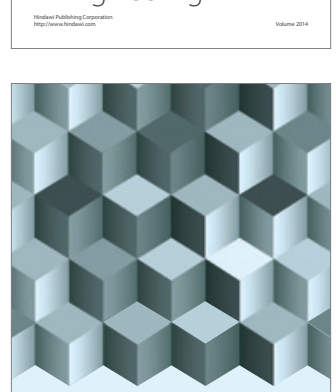

Journal of

Function Spaces
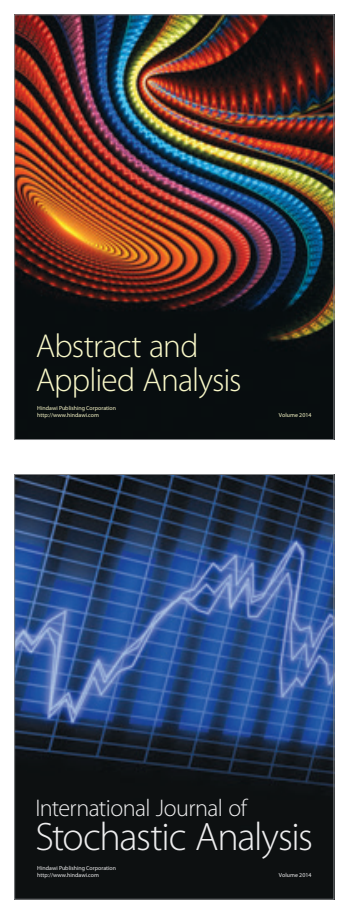

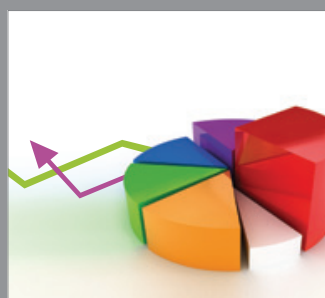

ournal of

Probability and Statistics

Promensencen
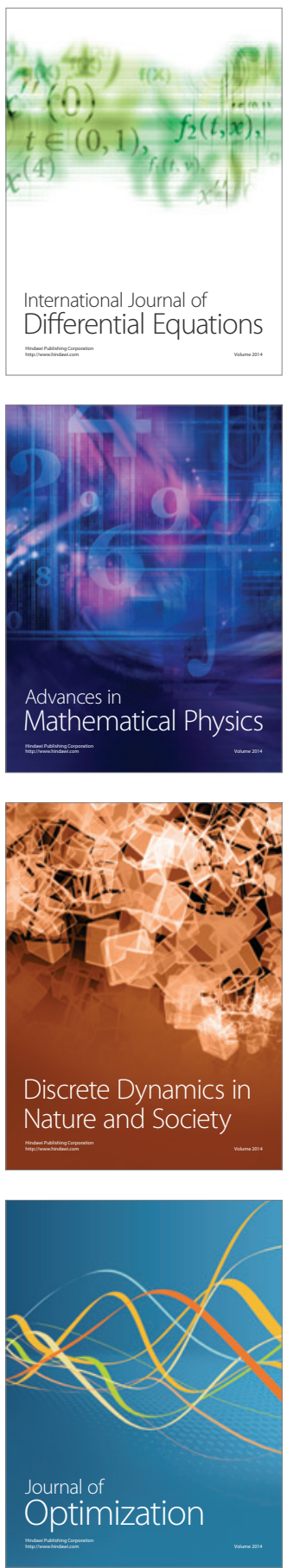